\title{
The Cornell-SLAC Pixel Array Detector at LCLS
}

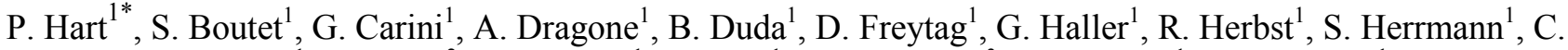 \\ Kenney ${ }^{1}$, J. Morse ${ }^{2}$, M. Nordby ${ }^{1}$, J. Pines ${ }^{1}$, N. van Bakel ${ }^{3}$, M. Weaver ${ }^{1}$, G. Williams ${ }^{1}$
}

\begin{abstract}
The Cornell-SLAC pixel array detector (CSpad) is a general-purpose integrating hybrid pixel $x$-ray camera developed for use at the Linear Coherent Light Source (LCLS) $x$-ray free electron laser at the SLAC National Accelerator Laboratory (SLAC). The detector has a full well capacity of about $2.5 \mathrm{k}$ photons in low-gain mode and a $S / N$ of about 6 in high-gain mode. Its $2.3 \mathrm{M}$ pixels are read out at $120 \mathrm{~Hz}$. The detector comprises $32500 \mu \mathrm{m}$ silicon sensors bump-bonded to $64185 \times 194-$ pixel ASICs. The pixel size is $110 \mu \mathrm{m}$. The water-cooled detector quadrants can be radially moved in-situ to vary the beam aperture. SLAC has built, calibrated, and optimized three complete camera systems based on a sensor and ASIC designed by Cornell. The camera is read out by a DAQ system which provides extensive online monitoring and prompt analysis capabilities. We have also built a dozen smaller cameras in a portable form-factor for use in confined spaces and for ease of development, testing, and deployment. Through 2012 user experiments have taken almost a petabyte of data with these detectors in a variety of applications. We have extensively tested the detector at synchrotrons and with an $x$-ray tube, in addition to commissioning tests at the LCLS, investigating linearity, crosstalk, homogeneity, and radiation hardness. The SLAC detector group is deploying improved support infrastructure and an updated ASIC and electronics based on this experience. This paper describes the instrument, its calibration and performance, and presents preliminary results from the updated camera.
\end{abstract}

\section{INTRODUCTION}

$\mathrm{T}$ HE LCLS provides $10^{12}$ photon pulses lasting only tens of femtoseconds at $120 \mathrm{~Hz}$. To exploit the wide variety of science the light source makes possible in the hard x-ray regime (mostly around $8-9 \mathrm{keV}$ but extending to around $24 \mathrm{keV}$ ) requires a large area detector with good resolution and large dynamic range capable of single-photon counting. A tiled hybrid pixel architecture with a single-slope 14-bit ADC was chosen to meet the stringent design requirements. The basic element is called the $2 \times 1$ and consists of a $21 \mathrm{~mm}$ by $43 \mathrm{~mm}$ by $500 \mu \mathrm{m}$ silicon sensor bump-bonded to a pair of ASICs. The sensor and the original version of the ASIC were designed by Cornell [1]. The pixel size is $110 \mu \mathrm{m}$. A pair of $2 \times 1$ modules are bonded to a rigid-flex carrier board and biased and read out by an analog and digital PCB. Signals and data are passed over LVDS lines or fiber, depending on the camera format. In some formats the sensor modules are

Manuscript received November 16, 2012. SLAC-PUB-15284.

${ }^{1}$ P. Hart ${ }^{*}$, G. Carini, A. Dragone, B. Duda, D. Freytag, G. Haller, R. Herbst, S. Herrmann, C. Kenney, M. Nordby, J. Pines, and M. Weaver are with the Research Engineering Division, SLAC National Accelerator Laboratory, Menlo Park, CA 94025 USA (*corresponding author, telephone: 650-926-2813, email: philiph@slac.stanford.edu). S. Boutet and G. Williams are at LCLS at SLAC.

${ }^{2}$ J. Morse is with the European Synchrotron Radiation Facility, 38043 Grenoble Cedex, France, and was on leave at SNAL for this work.

${ }^{3} \mathrm{~N}$. van Bakel was with SLAC and is now with the Detector R\&D Group at NIKHEF, Science Park 105, 1098 XG Amsterdam, Netherlands. mounted on movable quadrant plates, allowing the square beam aperture to be changed from $1 \mathrm{~mm}$ to $9 \mathrm{~mm}$. This is useful to protect the modules during alignment of the beam and to optimize the angular acceptance. Within the acceptance the camera is $86 \%$ active. The data is read out by a DAQ system which provides extensive display, monitoring, and analysis support. The detector is electronically shuttered with a $\sim 10 \mu$ s window, largely eliminating sensitivity to leakage current and temperature variation and can thus run at room temperature, which simplifies installation and operation. The camera uses power pulsing to reduce the heat load, which is about $50 \mathrm{~W}$, and is temperature-controlled using flexible cold straps or direct mounting to a water-cooled plate.

The camera was originally developed for use at the Coherent X-ray Imaging (CXI) instrument [2, 3, 4] at LCLS, but is also the principal detector at the Matter In Extreme Conditions instrument [5] and is in regular use at the X-ray Pump Probe (XPP) instrument [6], where it was first used in 2010. The camera has been used at the X-ray Coherent Scattering instrument as well as at the Stanford Synchrotron Radiation Lightsource and at the Argonne Advanced Photon Source, and will soon be used for an experiment at SACLA.

\section{2.3MPIXEL CAMERA DESCRIPTION}

For a complete description, see [7]. The sensor is made of ntype, float-zone silicon with a resistivity greater than 5,000 Ohm-cm and is fabricated by SINTEF. See Fig. 1. The sensitive area of approximately $20.3 \mathrm{~mm}$ by $43.0 \mathrm{~mm}$ has 185 by 388 pixels. The pixel diffusions are $\mathrm{p}$ type and collect holes. The entrance face is an $\mathrm{n}+$ phosphorus diffusion covered by a $1.2 \mu \mathrm{m} \mathrm{Al}$ layer. The wider, inner $\mathrm{p}+$ guard ring is connected to the circuit-chip ground. There are about $400 \mu \mathrm{m}$ of insensitive area between the edge pixel and the saw cut. The $500 \mu \mathrm{m}$-thick sensors deplete around $120 \mathrm{~V}$ and are operated at $190 \mathrm{~V}$.
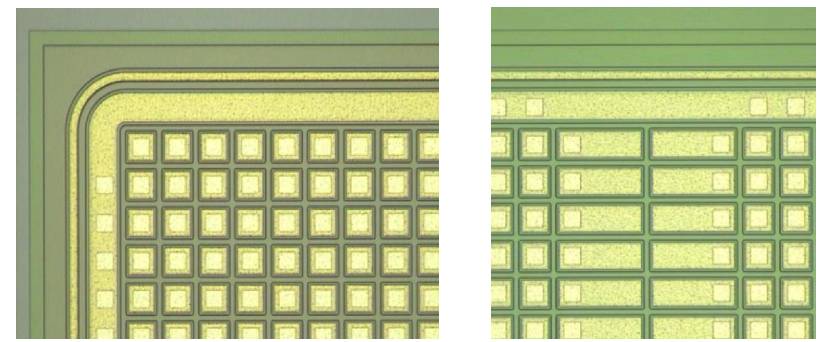

Fig. 1. Left, the corner of the sensor, showing the 185 by $193110 \mu \mathrm{m}-$ square pixel arrangement and guard rings. Right, the region covering the inter-asic boundary, showing the two rows of $275 \mu \mathrm{m}$-long pixels there.

The ASIC pixel architecture is shown in Fig. 2. The DCcoupled input amplifier has two gain settings with a ratio of $7: 1$. The gain bit in each pixel can be set independently. After a sample-and-hold a comparator fires when an external ramp 
falls below the signal voltage, stopping a counter. The amplifier and comparator are controlled by four externallysupplied bias currents. The ASIC, which is made at TSMC, is organized into seven blocks of 26 columns and one of 12 .

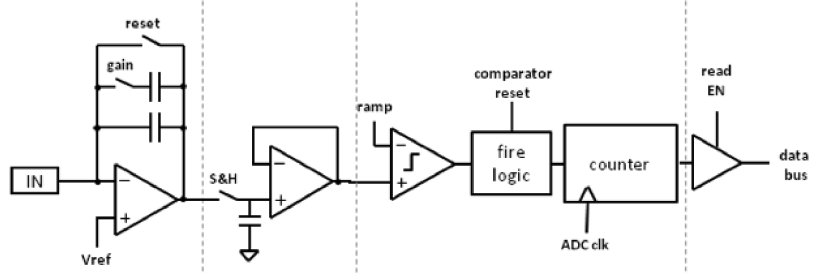

Fig. 2. Schematic representation of the ASIC pixel electronics.

A group of 16 ASICS is supported by an analog and a digital PCB. The analog board provides the ramp and biases. The digital board is based on a Virtex-5 FPGA, controlling the ASIC and sending the data downstream via a $625 \mathrm{Mbit}$ serial link.

After the sensor and ASIC are bump-bonded, they are bonded to a gold-plated polyimide carrier board using Nusil adhesive, which is vacuum compatible and provides good thermal contact. The ASICs are wire-bonded to gold pads on the carrier board. The board incorporates a Kapton flex cable to which a high-density 92-pin connector is soldered; the flex exits from the bottom of the rigid carrier not the edge, making denser integration possible. After testing, a pair of $2 \times 1$ modules is bonded to an Al carrier plate. See Fig. 3.

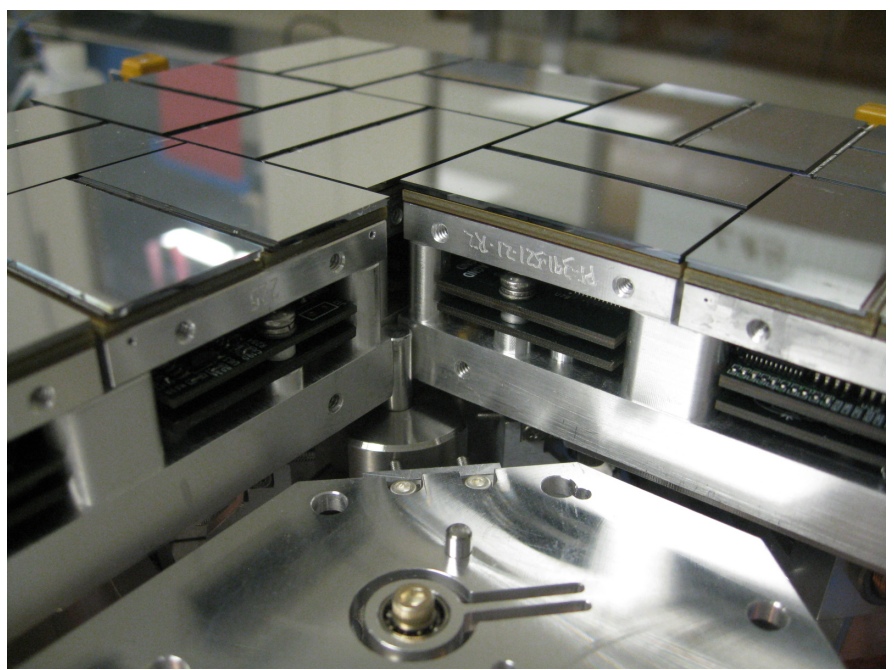

Fig. 3. The camera in construction, showing three of four quadrants.

The quadrants are mounted on $\mathrm{Al}$ base plate assemblies as seen in Fig.4. In the CXI versions of the camera, linear crossroller bearings from Del-Tron and custom flexures are driven by a Newport UHV stepper motor to vary the beam aperture with precision. Flexible $\mathrm{S}$-shaped $\mathrm{Cu}$ cold straps connect the quadrant plates to a water-cooled $\mathrm{Cu}$ plate; the modules are cooled via bosses passing through the PCBs. Thermistors on the module carriers and on the quadrant plates are read out by the DAQ and slow-control system. A cylindrical shroud is mounted to the front of the detector to support an $8 \mu \mathrm{m}$-thick $200 \mathrm{~nm}$-aluminized polyimide sheet. For some experiments a grid of $0.5 \mathrm{~mm} \mathrm{~W}$ wires is mounted in front of the detector face for alignment and to provide shadowed pixels.

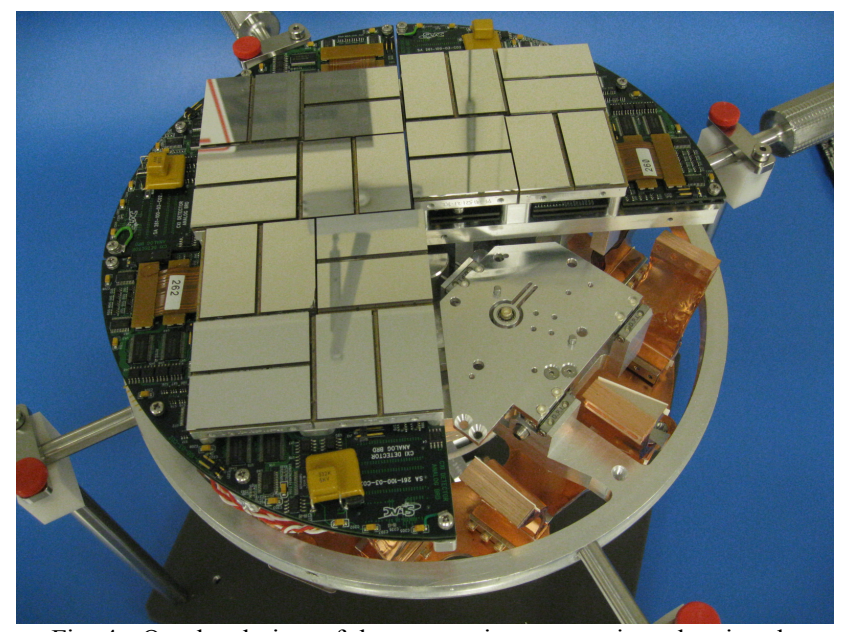

Fig. 4. Overhead view of the camera in construction, showing three of four quadrants.

The data is read out over a custom protocol into the DAQ system. The DAQ aligns information from each camera frame with accelerator, controls, and other instrument information from the same FEL pulse. It provides an online display and analysis suite, allowing the user to apply dark and frame noise corrections and mask hot pixels; to integrate in $\mathrm{x}-\mathrm{y}$ or $\mathrm{r}-\mathrm{phi}$ regions; to display noise, average value, or photons selected using noise and gain maps. The user can also plug in modules to filter the display or to plot more sophisticated analysis variables. See Fig. 5.

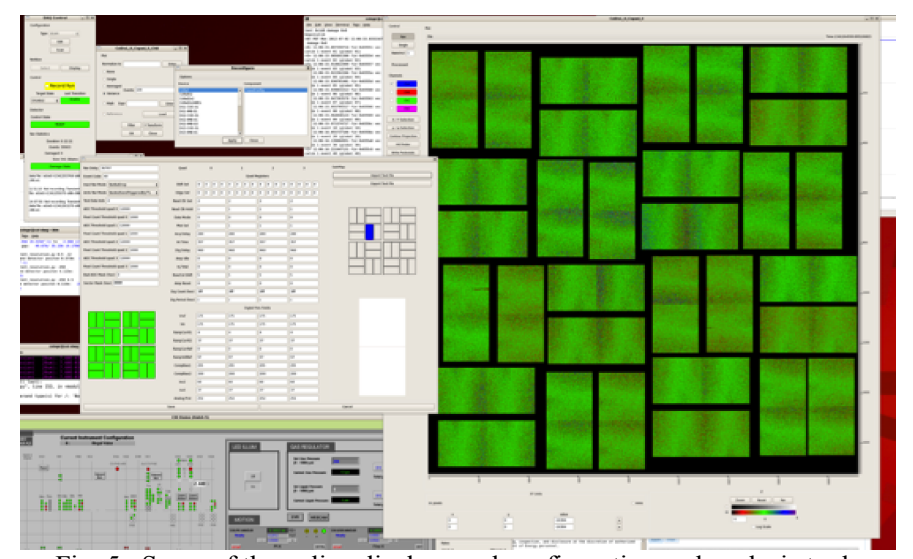

Fig. 5. Some of the online displays and configuration and analysis tools for the CSPAD.

SLAC has built three of these large cameras, comprising about 7.3Mpixels.

\section{140KPIXEL VERSION}

In 2012 we began deploying a smaller version of the camera with two sensors, optional Peltier cooling, and fiber readout. See Fig. 6. The compact camera head allows this version to be used in confined spaces in novel arrangements of up to eight cameras simultaneously so far. The geometry also greatly simplifies debugging by exposing the electronics, shortening 
the development cycle. Studies on the $140 \mathrm{k}$ have provided useful insight into the detector behavior and yielded improved electronics and operating configuration. The use of an analog reset scheme and improved biasing results in a quieter, more homogenous response. See $[8,9]$ for further details.

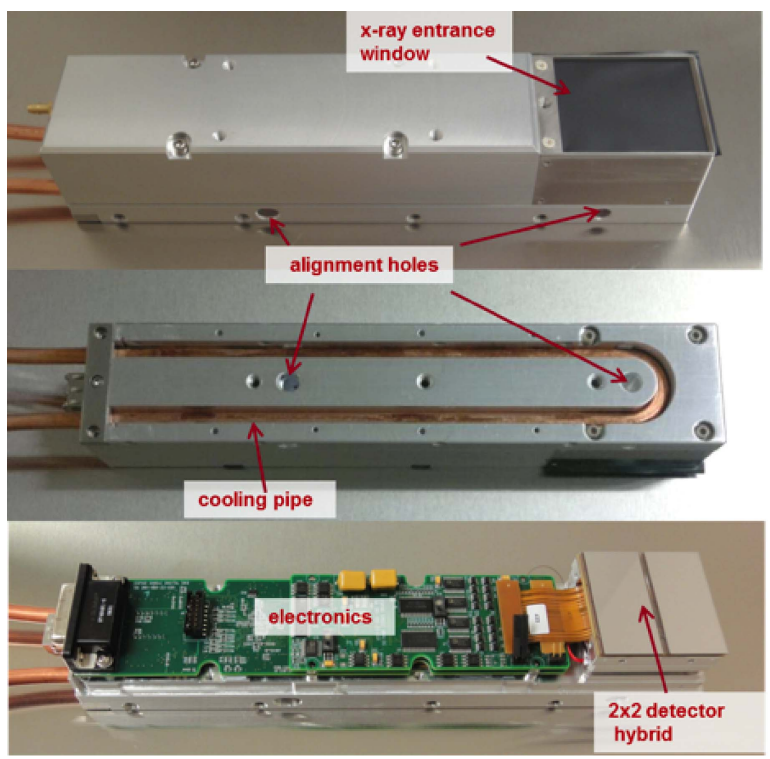

Fig. 6. Views of the $140 \mathrm{~K}$ version of the camera. Another form-factor, with the sensor mounted on the long axis of the camera, is also in use.

\section{CAlibration AND PERFormance}

The camera has been calibrated with darks and flat field illuminations in-situ and/or with an x-ray tube. The gain of each pixel is determined by fitting the single-photon peak from $\mathrm{Cu} \mathrm{K} \alpha$, the slope of the measured signal as a function of beam attenuation, or comparison of the signal distribution in water scattering against known models. The gain typically varies by about $20 \%$ across the ASIC. The detector is linear at below the $0.2 \%$ level in tests at synchrotrons over most of the dynamic range up to saturation. See Fig. 7.

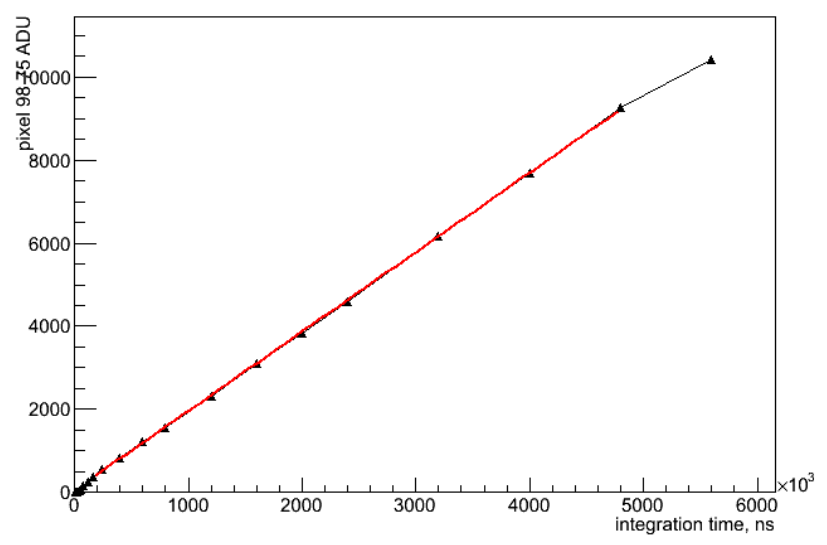

Fig. 7. The 2.3Mpixel camera response in high gain as a function of input. Data taken at SSRL B2-2.

For reasons described further in [8, 9], there is coupling between pixels with similar input levels (including pedestal value) in such a way that the pixel comparator is more likely to fire if other comparators on the ASIC have recently done so. The single-slope ADC architecture converts signal voltage to time, so it can be seen that high- and low-pedestal pixels, which fire early and late, respectively, have the design gain, while those with pedestals near the mode have low gain and noise. See Fig. 8. This can be corrected by calibration when the signal exposure across a module is uniform, but complicates the detector behavior when both low- and high-range signals are important.

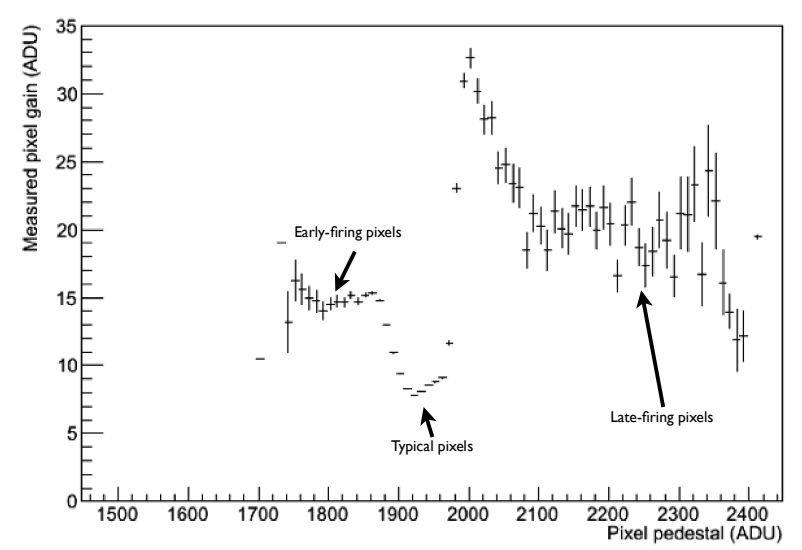

Fig. 8. The measured gain from the single-photon peak as a function of pixel pedestal, showing that pixels in the core of the pedestal distribution are coupled.

The detector has proven to be stable. There is about 3 ADU (RMS) frame noise, about a third of a photon in the original version of the camera. This shift is fairly flat across the sensor, allowing it to be removed in most low-flux cases (at higher flux it can simply be ignored) by using pixels intentionally left unbonded, shadowed pixels, or by histogramming all pixel values after dark correction and identifying the first peak on the left as pixels without signal. The camera is sensitive to temperature variation at about $-12 \mathrm{ADU} / \mathrm{C}$, but temperature variation during a run is typically well below $1 \mathrm{C}$ and this is below the noise level, especially after frame noise correction.

The detector has proven to be sufficiently radiation hard for several-year operation at least. In early running it was found that the portion of the ASIC not covered by the sensor is vulnerable to damage, rendering the chip unusable, so we have installed $50 \mu \mathrm{m} \mathrm{W}$ foil shields over that area. This has been successful at protecting the ASICs. There is also local damage to individual pixels, but the rate of such damage has greatly decreased at CXI following the installation of a mirror system which cuts off the higher beam harmonics. See [10] for a discussion of the foils and radiation damage studies.

While the dynamic range is as expected in most circumstances, we have seen that under some very high-flux saturating conditions at LCLS the detector response is less than the normal saturation level. This has not been observed in synchrotron running, perhaps indicating an FEL-specific issue. This effect must be investigated, as it is significant for planned higher-dynamic-range detectors.

The precise pixel positions within a quadrant (and within the entire XPP camera array) are determined by optical metrology. 
Relative quadrant positions for the cameras with movable quadrants are determined to about a pixel by shadows from the wire masks, diffraction rings, and motor encoding.

\section{UPGRADES}

In the $140 \mathrm{k}$ version of the camera improved electronics reduces the coupling over the ASIC biasing. We have updated the analog quadrant PCBs for the downstream camera at CXI using the same approach. The new circuitry uses a time-variant filter to supply the external biasing to the ASIC and at the same time allows for power cycling the bias nodes and therefore the complete ASIC. The time-variant filter uses an RC filter with a switched capacitor to attenuate disturbance on the bias nodes. By opening the switch and removing the capacitor from the bias node we can power cycle the ASIC quickly.

We have also built an updated version of the ASIC, including improved power distribution, internal biasing and power cycling, and a more stable comparator design. The effect on noise homogeneity is shown in Fig. 9. The effect shown in Fig. 8 is now almost entirely gone.
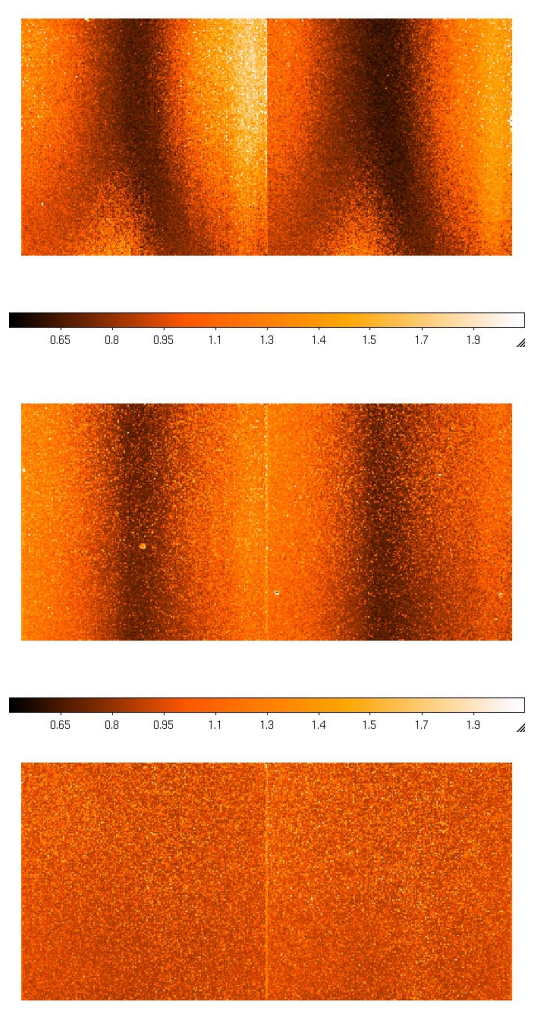

Fig. 9. Top, the relative noise across a detector module with the original electronics and ASIC. Center, a module with updated electronics. Bottom, a fully updated module.

\section{CONCLUSION}

SLAC has built and operated three large CSPAD cameras over the last three years, and over the last year introduced a smaller, flexible version. We have made significant progress in calibrating and understanding the behavior of the detector and are steadily improving its performance. Almost a petabyte of data has been recorded with CSPAD cameras. The SLAC detector and ASIC groups are applying the experience gained to build new cameras [11] for LCLS and LCLS-II.

\section{ACKNOWLEDGMENT}

This work is supported by the Department of Energy, Laboratory Directed Research and Development funding, under contract DE-AC02-76SF00515. Portions of this research were carried out at the Linac Coherent Light Source and the Stanford Synchrotron Radiation Lightsource, National User Facilities operated by Stanford University on behalf of the U.S. Department of Energy (DOE), Office of Basic Energy Sciences (BES). We thank the many staff members at SLAC who have supported this work, in particular Lupe Salgado. We especially thank Sol M. Gruner, Hugh Philipp, Lucas Koerner, Marianne Hromalik, and Mark Tate at Cornell University for the design and development of the sensor and ASIC.

\section{REFERENCES}

[1] Hugh T. Philipp, Marianne Hromalik, Mark Tate, Lucas Koerner, Sol M. Gruner. "Pixel array detector for x-ray free electron laser experiments". Nucl. Instr. Meth. Phys Res. A. (2011) 649, 67-69

[2] S. Boutet and G. J. Williams, "The Coherent X-ray Imaging (CXI) Instrument at Linac Coherent Light Source (LCLS), New Journal of Physics, 12 (2010)

[3] S. Boutet et. al., "High-Resolution Protein Structure Determination by Serial Femtosecond Crystallography", Science, Vol 337 no. 6092 pp. 362-364 (2012)

[4] J. Kern. A. Alonso-Mori, J. Hellmich, U. Bergmann, J. Yano, V. Yachandra, et al., "Room temperature X-ray diffraction of photosystem II microcrystals", Proceedings of the National Academy of Sciences, June 29, 2012, vol. 109 no. 25 9721-9726

[5] http://lcls.slac.stanford.edu/mec/

[6] D. Fritz, "The X-Ray Pump-Probe Instrument at LCLS," in Laser Science XXV, OSA Technical Digest (CD) (Optical Society of America, 2009), paper LSMD2.

[7] P.Hart, S. Boutet, G. Carini, et al. "The CSPAD megapixel x-ray camera at LCLS", Proc. SPIE 8504, X-Ray Free-Electron Lasers: Beam Diagnostics, Beamline Instrumentation, and Applications, 85040C (October 15, 2012)

[8] Sven Herrmann et al., "CSPAD-140k - a Versatile Detector for LCLS Experiments", NIMA proceedings on the 12th Pisa Meeting on Advanced Detectors, 2012

[9] S. Herrmann, S. Boutet, G. Carini, A. Dragone, D. Freytag, G. Haller, P. Hart, R. Herbst, C. Kenney, J. Pines, G. Williams,"CSPAD-140k Experimental Applications at LCLS", to appear in NSS-MIC 2012 conference records.

[10] A. Tomada, S. Boutet, B. Duda, P. Hart, C. Kenney, L. Manger, M. Messerschmidt, J. Tice, G. Williams, "High-Z Radiation Shields for Xray Free Electron Laser Detectors", to appear in NSS-MIC 2012 conference records.

[11] A. Dragone, P. Caragiulo, D. Freytag, P. Hart, R. Herbst, S. Herrmann, C. Kenney, J. Segal and G. Haller, "eLine100: A Front End ASIC for LCLS Detectors in Low Noise Applications", to appear in NSS-MIC 2012 conference records. 